\title{
Modeling Household Transmission of American Trypanosomiasis
}

\author{
Joel E. Cohen ${ }^{1 *}$ and Ricardo E. Gürtler ${ }^{2}$
}

\begin{abstract}
American trypanosomiasis, or Chagas disease, caused by the protozoan parasite Trypanosoma cruzi and transmitted by blood-feeding triatomine bugs, is a chronic, frequently fatal infection that is common in Latin America. Neither adequate drugs nor a vaccine is available. A mathematical model calibrated to detailed household data from three villages in northwest Argentina shows that householders could greatly reduce the risk of human infection by excluding domestic animals, especially infected dogs, from bedrooms; removing potential refuges for bugs from walls and ceilings; and using domestically applied insecticides. Low-cost, locally practicable environmental management combined with intermittent use of insecticides can sustainably control transmission of $T$. cruzi to humans in rural Argentina and probably elsewhere.
\end{abstract}

Chagas disease, or American trypanosomiasis, is endemic in Central and South America. An estimated 16 to 18 million persons are infected with Trypanosoma cruzi (Kinetoplastida: Trypanosomatidae), the causative agent of Chagas disease, and 100 million people (roughly one-quarter of the population) are at risk of infection (1). Despite decreasing rates of prevalence and incidence of $T$. cruzi infection $(2,3)$, Chagas disease remains a serious obstacle to health and economic development in Latin America, especially for the rural poor.

The repertoire of control measures is limited. Two drugs are curative in the acute and early chronic phase of infection but have adverse effects and may not always eliminate $T$. cruzi. No vaccines are available to prevent infection. Transmission may be interrupted by residual spraying to kill blood-feeding triatomine bugs (the vector of $T$. cruzi), screening blood donors, and treating infected infants born to infected mothers. A more controversial strategy for interrupting transmission is to divert bugs from humans by the use of animals that are not susceptible to T. cruzi (4). This strategy, called zooprophylaxis, is controversial for other vector-borne diseases (5) as well and may remain so for $T$. cruzi because it cannot be tested experimentally. Ethical considerations bar a randomized prospective field study in which the human prevalence of $T$. cruzi infection is compared between households that do, and those that do not, keep domestic animals in bedroom areas in the presence of domestic triatomine infestations.

'Laboratory of Populations, Rockefeller University and Columbia University, 1230 York Avenue, Box 20, New York, NY 10021, USA. '2Laboratorio de Ecología General, Facultad de Ciencias Exactas y Naturales, Universidad de Buenos Aires, Ciudad Universitaria, C1428EHA Buenos Aires, Argentina.

*To whom correspondence should be addressed. Email: cohen@rockefeller.edu
Mathematical models of the transmission of $T$. cruzi infection are the next best available tool to understand the effects of alternative control strategies (6-14) [Web supplement gives further references (15)]. Here we present a model of the transmission of $T$. cruzi infection within an individual household. The model represents three vertebrate populations (humans, dogs, and chickens), the bug population, the parasite, and seasonality. Although many existing models of the transmission of infectious diseases use differential equations to represent changes in the prevalence of infection and in the population sizes of hosts and vectors, which are usually assumed to be large, the discrete formalism of the present model makes it easy to represent the age structure of one household's human population, the small numbers of domestic vertebrates, and the seasonal differences in host composition and exposures to bugs.

This model was developed in close connection with household data collected to support modeling in three rural villages, Amamá, Trinidad, and Mercedes, in the province of Santiago del Estero, northwest Argentina. The villages are situated within $9 \mathrm{~km}$ of each other in a semi-arid hardwood thorny forest habitat. In 65 houses of Amamá and Trinidad, the median household in 1993 had five people, about three infected dogs, no more than one cat, and 8 to 27 chickens and ducks (16).

In Argentina, transmission of T. cruzi to humans is minimal in fall and winter (April to August). Below $16^{\circ}$ to $18^{\circ} \mathrm{C}$, bugs cease development and feeding (17). In early spring, people sleep indoors, chickens are maximally present in bedroom areas (18), bugs are increasingly active and feeding, and the domiciliary bug population grows rapidly. In summer, chickens mainly roost outdoors, people usually move their raised beds outdoors to sleep on verandas or patios in front of their bedrooms, and the size of the domiciliary bug population is maximal.

Domestic triatomine bugs take blood meals from household vertebrates to be able to develop from each instar to the next and to lay eggs. Keeping chickens in bedrooms in spring to protect them or their eggs from predation or theft increases the domestic bug population size (18, 19 ), most notably in the following summer. Because chickens cannot be infected with $T$. cruzi, the more often a domestic bug feeds on a chicken, the less likely the bug is to become infected with $T$. cruzi. Therefore, keeping chickens in bedrooms could decrease the prevalence rate of $T$. cruzi in bugs. In contrast, keeping infected dogs in the household increases both the bug population size (20) and bug prevalence of $T$. cruzi $(21,22)$. The summer population of large and late-stage $T$. infestans bugs, increased as a result of spring feeding on chickens, shifts feeding from chickens to humans or infected dogs in the hot summer season (16) when chickens are largely absent. The presence of chickens in bedroom areas decreased the prevalence of $T$. cruzi in bugs but increased the density of $T$. cruzi-infected bugs (23).

Mathematical modeling is required to understand the implications of these findings for the human prevalence of $T$. cruzi infection (4). We model only the transmission season, spring (September to mid-December) and summer (mid-December to March). The model represents the population of the parasite $T$. cruzi implicitly through prevalence rates. The human (or dog) prevalence rate gives the proportion of humans (or dogs) who are infected with $T$. cruzi (as measured by seropositivity) but does not distinguish among different phases of infection. In these calculations, all dogs are assumed to be infected, as field data show. The bug prevalence rates (in spring and in summer) give the fraction of bugs in each season that are infected with $T$. cruzi. In the model and real life, once a person, dog, or bug becomes infected, that individual remains infected for life (unless the person or dog is treated promptly; there are no treatments for bugs).

The model represents explicitly four populations: humans, dogs, chickens, and bugs. Humans are represented by the numbers of individuals in 5-year age groups: under age 5, ages 5 to 9 , ages 10 to 14 , etc. Dogs represent all mammalian domiciliary animals that are susceptible to infection with $T$. cruzi, provide blood meals to T. infestans, and are more attractive or accessible as a source of blood meals than humans. Chickens represent all avian domiciliary animals, which are not susceptible to infection with $T$. cruzi but which may provide blood meals to $T$. infestans and are more attractive or accessible blood sources than humans. In the model and henceforth here, "bugs" means exclusively fourth- and fifth-instar nymphs and adults. These stages include almost 


\section{REPOR T S}

all bugs that are infected $(23,24)$ and capable of transmitting infection, and timed manual collections of bugs yield samples biased toward these stages.

Bugs are several times more likely to take their blood meals from dogs and chickens than from humans (20). For example, the ratio of dog blood meals to human blood meals in the engorged guts of bugs was 2.3 to 2.6 times the ratio of the number of dogs to the number of humans in a household in the spring-summer period. Similarly, bugs selected chickens for blood meals nearly five times as often as the number of chickens relative to humans. These ratios did not vary significantly among households with differing total numbers of humans, dogs, and chickens (20). Dogs and chickens are probably less attentive than humans in defending themselves against feedings by the bugs. Also dogs and chickens sleep or nest in bedroom places that are more accessible to the bugs than the raised beds on which people typically sleep, whether indoors or outdoors. We approximate the ratio of feedings on dogs and chickens relative to humans as 3 , as a rough midpoint of the large range of variation of empirical estimates. The probability of infecting an initially uninfected bug in one full blood meal from an infected dog was 12 times that probability from infected children and 200 times that from infected adults (10).

The assumptions, variables, and formal structure of the model are detailed in the Web supplement (15). The model predicts how the numbers of humans, chickens, and infected dogs in a household and the physical-chemical conditions affect the prevalence of $T$. cruzi infection in humans and bugs, the number of infected and uninfected bugs, and the distribution of feeding contacts, by season. Only one parameter of the model was varied freely to improve the quantitative fit between observation and prediction: the probability of transmission of infection from an infected bug to an uninfected person $\left(t_{B \rightarrow H}=0.0008\right)$. This value is close to the 0.0009 estimated from one field study (9). For obvious ethical reasons, this probability cannot be estimated experimentally and is largely unknown.

Given the complexity of the natural system and the relative simplicity of any mathematical model, qualitative agreement is the most that can be hoped for from the comparisons of model predictions and field observations. The following comparisons suggest that the qualitative predictions of the model give some insights into the real household transmission of T. cruzi.

Empirically (Fig. 1A) and theoretically (Fig. 1B), the bug prevalence rate increased rapidly with the fraction of bugs that took blood meals from infected dogs (or cats, which were relatively rare) when the fraction of bugs that took blood meals from chickens stayed constant. The bug prevalence rate de-

A creased slowly with an increasing fraction of bugs that took blood meals from chickens when the fraction of bugs that took blood meals from infected dogs stayed constant.

Empirically (Fig. 1C) and theoretically (Fig. 1D), as the relative density of bugs collected per unit of search effort at the end of summer increased, the fraction of bugs that contained blood meals from humans decreased while the observed fraction of bugs that contained blood meals from chickens increased. The model predictions are shown for spring and summer combined because blood meals from both seasons were probably detectable in bugs collected at the end of summer (16). The source of a full
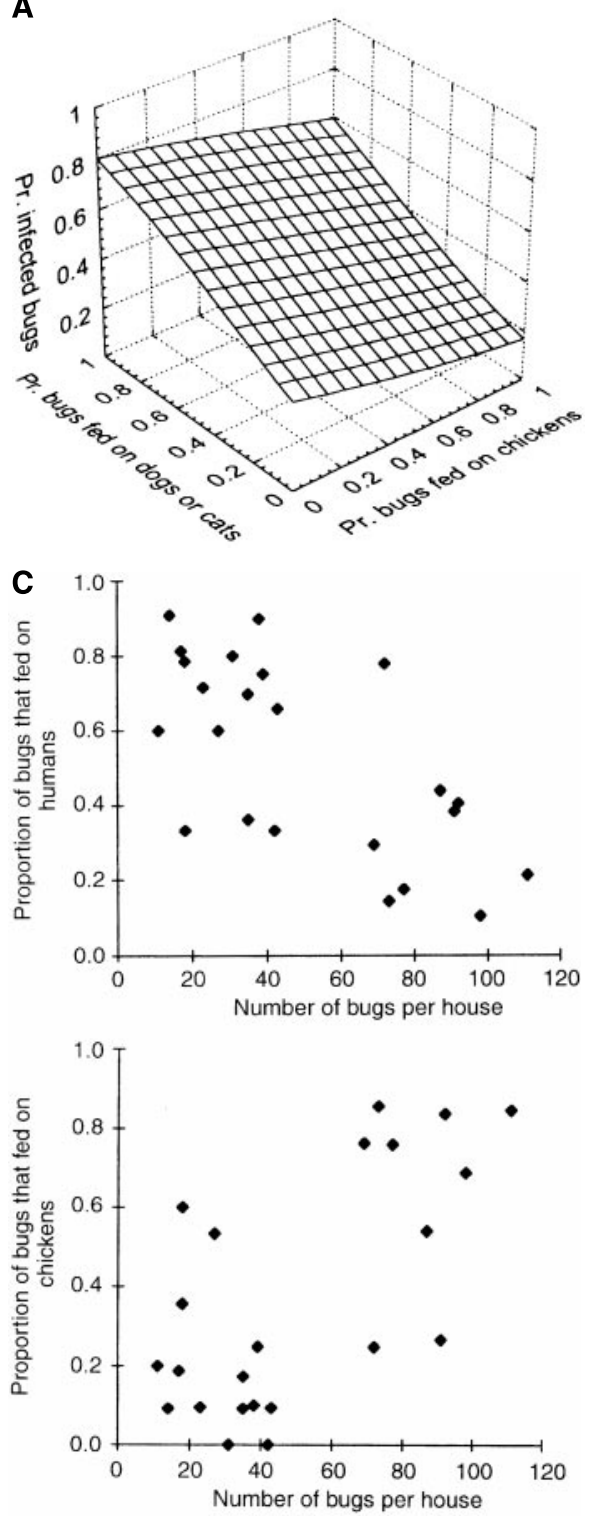
may have had feeding contacts with more than
one species of vertebrate. A given bug may be counted more than once in $(A)$, but each feeding contact counts only once in (B). (C) Fraction of bugs that contained blood meals from humans (top) and chickens (bottom) as a function of the bug density per unit of searching effort. Reproduced by permission from Fig. 2, a and b, in (20), p. 706. Each point belongs to a different household. (D) Predicted fractions of feeding contacts on humans (o) and chickens $(x)$ in spring and summer (combined because of the carryover of blood meals) as a function of the predicted number of summer bugs for all combinations of numbers of dogs and chickens considered.

blood meal taken at an earlier instar is detectable for up to 3 months from blood taken from the gut of a later instar of the same individual bug. The intuitive explanation as to why both field data and the model show that increasing number of summer bugs are associated with fewer human feeding contacts and more chicken feeding contacts is that, for any given number of dogs, the summer bug population increases as a result of increasing availability of chickens in spring, and chickens are more accessible or attractive than humans as blood meal sources for the bugs.

The two principal predictions of the model are as follows:

B
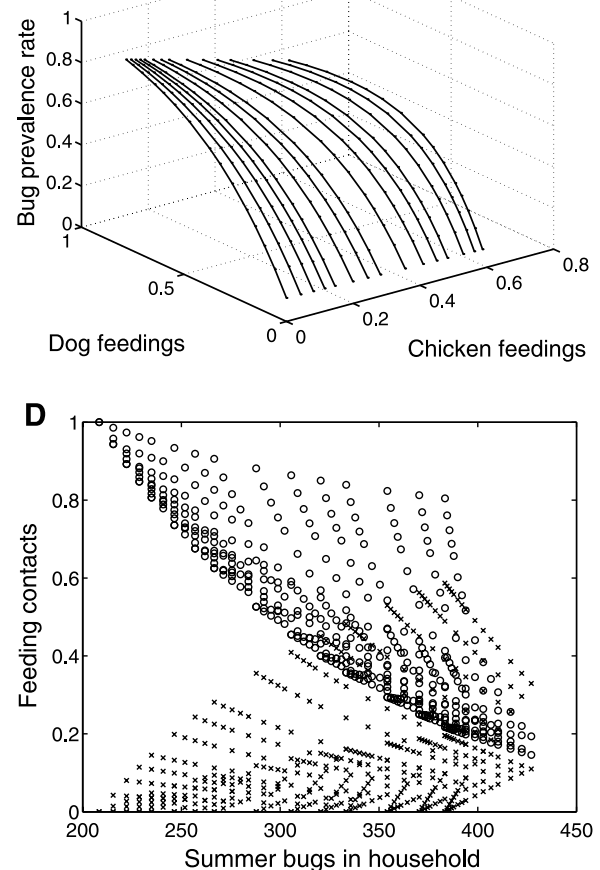

Fig. 1. Comparison of observations (left) made in March (end of summer in Southern Hemisphere) from three rural villages in Santiago del Estero, northwest Argentina, with model predictions (right). (A) Bug prevalence rate as a function of the fraction of bugs that contained blood meals from dogs (and cats, which were much less frequent) and from chickens. A logistic regression surface was fitted to data on individual households. Reproduced by permission from Fig. 4a in (23), p. 754. (B) Predicted summer bug prevalence rate as a function of the predicted fractions of spring feeding contacts with dogs and chickens. Although each feeding contact had cherch 


\section{R E P O R T S}

1) The worst thing householders can do, from the point of view of limiting $T$. cruzi prevalence, is to keep roughly two infected domestic dogs (Fig. 2I). This is precisely what many households do. The intuitive explanation why the model predicts that roughly two infected dogs should be pessimal for human prevalence is that each dog is as attractive as three humans, and five humans are assumed in the household, so two dogs is roughly equivalent to the five humans from the bugs' point of view. As the number of infected dogs increases up to two, the infected dogs are an increasing and highly infective source of $T$. cruzi. As the number of infected dogs increases beyond two, the additional infected dogs contribute infection to many already infected bugs but also divert proportionally more of the feeding bugs from humans. This intuitive account explains not only the location of the peak human prevalence rate around two infected dogs, but also explains why the rise of human prevalence from zero to two infected dogs is faster than the very gradual fall of human prevalence as the number of infected dogs increases beyond two.

2) Elimination of infected dogs from a household with infected people is nearly sufficient to extinguish transmission of $T$. cruzi, barring reintroduction of infected dogs, children, or bugs (Fig. 2, E, F, G, and I).

The model makes many additional predictions (Fig. 2).

3.1) The number of spring bugs increases with the number of dogs but is independent of the number of chickens (Fig. 2A). Chickens, kept in the household in spring, have no immediate effect on spring bugs because the model assumes a lag of one season between the availability of blood sources and the recruitment of late-instar and adult bugs.

3.2) The number of summer bugs increases with both dogs and chickens in the previous spring (Fig. 2B), as 1988-1989 data at Amamá, Trinidad, and Mercedes showed (18-20).

3.3) The fraction of spring bugs' feeding contacts with humans (Fig. 2C) decreases symmetrically, rapidly at first and then more slowly, with more dogs and chickens.

3.4) The fraction of spring bugs' feeding contacts with infected dogs (Fig. 2D) increases at a diminishing rate with more infected dogs and decreases with more chickens. The fraction of spring bugs' feeding contacts with chickens (not shown) is obtained by exchanging the axes of Fig. 2D for chickens and dogs.

3.5) The fraction of spring bugs infected with $T$. cruzi (the spring bug prevalence rate) increases at a diminishing rate with more infected dogs and decreases slightly with more chickens (Fig. 2E). Even when there are no infected dogs and no chickens, the spring bug prevalence rate remains positive because infected people remain in the household (by assumption in the model, and because of the persistent presence of chronically infected adults in the field). When there are no dogs, an increase in the number of chickens diverts bugs from feeding on humans to feeding on unsusceptible chickens and gradually reduces the bug prevalence rate.

3.6) The prevalence rate in summer bugs (not shown) increases with the number of infected dogs as in Fig. 2E. Observed bug prevalence rates of $T$. cruzi infection increased with the number of infected dogs in 1988-1989 in Amamá (21). In Trinidad and Mercedes, the mean bug prevalence rates were $16,41,68$, and $47 \%$ in households with zero, one, two, and three infected dogs, respectively (22). The positive mean bug prevalence rate in households with zero infected dogs is due to the presence of infected cats. Bug prevalence rates were $4 \%$ in households with no infected dogs or cats and $35 \%$ in households with an infected cat and no infected dog.

3.7) The spring number of infected bugs (Fig. 2F) parallels the spring bug prevalence rate (Fig. 2E) because the spring bug population size is independent of the number of chickens (Fig. 2A).

3.8) For any fixed number of dogs, households with more chickens have slightly more infected summer bugs (Fig. 2G) and substantially more total summer bugs (Fig. 2B). Thus, the presence of chickens in bedrooms in spring decreases the number of infected bugs in spring and increases the number of
A
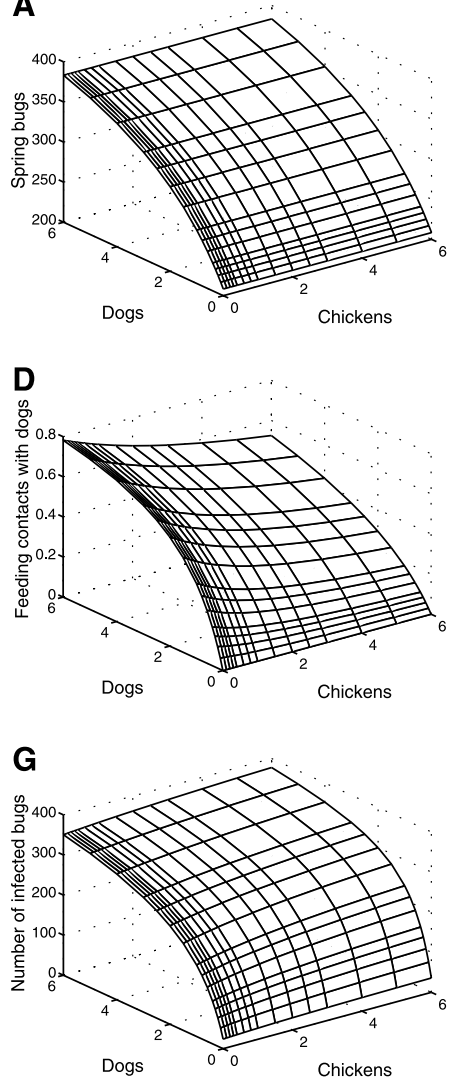

B

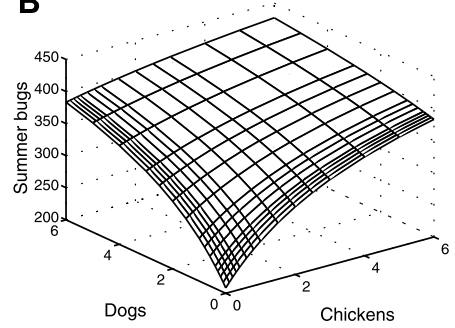

E
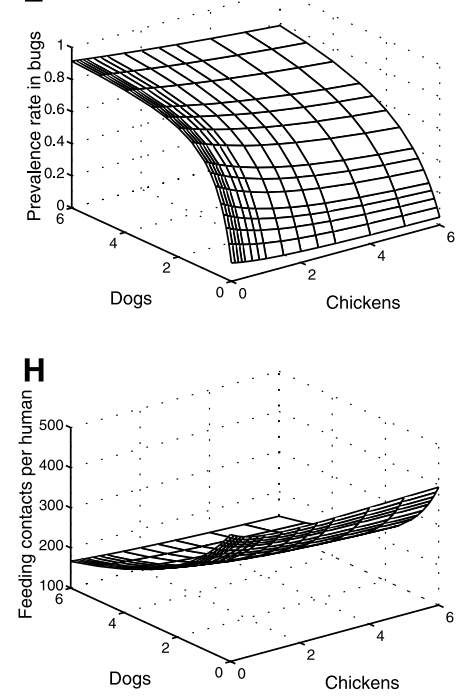

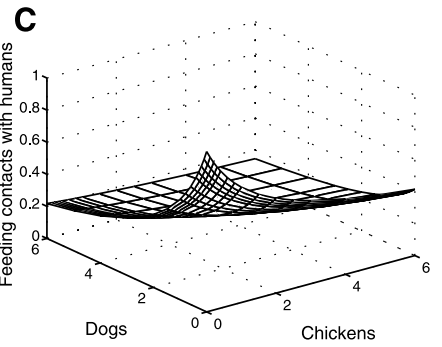

$\mathbf{F}$
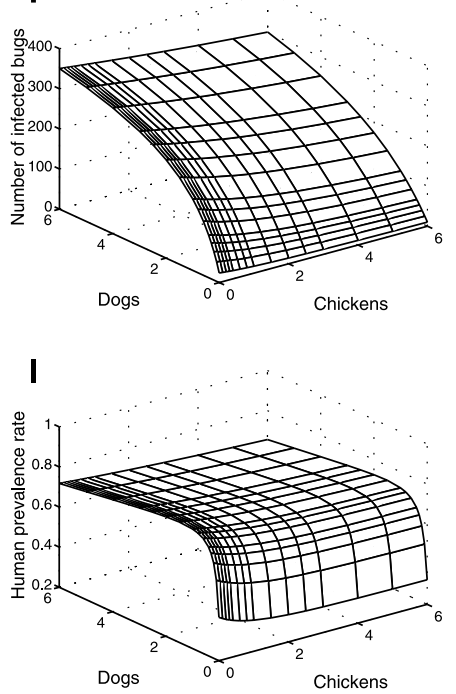

Fig. 2. Predictions from a model of the household transmission of $T$. cruzi for selected combinations of chickens and infected dogs. (A) Number of bugs in household in spring. (B) Number of bugs in household in summer. (C) Fraction of feeding contacts from humans in bugs captured in spring. (D) Fraction of feeding contacts from dogs in bugs captured in spring. (E) Prevalence rate in bugs captured in spring. (F) Number of infected spring bugs. (G) Number of infected summer bugs. (H) Number of feeding contacts per human per year. (I) Human prevalence rate, or fraction of humans infected, at steady state. 


\section{R E P O R T S}

infected bugs in summer. The summer increase in infected bugs is slightly smaller than the absolute spring decrease in infected bugs for any positive number of chickens in spring.

3.9) The number of feeding contacts per human per year (Fig. 2H) decreases rapidly with increasing numbers of dogs and decreases very slightly with increasing numbers of chickens, because the chickens divert feeding contacts only in spring.

3.10) The number of potentially infective feeding contacts per human per year is the product of the number of feeding contacts per human per year (Fig. 2H) times a weighted average of the spring (Fig. 2E) and summer bug prevalence rates. The number of potentially infective feeding contacts (not shown, but similar in shape to Fig. 2I) rises to a peak with an increase from 0 dogs to 1.5 infected dogs, and then begins to decline very slightly as further dogs are added to the household. (When the continuous variables used to measure the sizes of host or bug populations include fractions, the fractions may be interpreted as the average fraction of time individuals are present in the household.) With additional dogs, more bugs are diverted from feeding on humans because bugs prefer to feed on dogs.

3.11) The human prevalence rate, or fraction of humans infected (Fig. 2I), is positive even in the absence of chickens and dogs because infected humans are initially present in the house. For any fixed number of infected dogs, the human prevalence rate declines very slowly with more chickens. For any fixed number of chickens, the human prevalence rate increases very rapidly as the number of infected dogs increases from 0 to roughly 1.5 and then slowly declines with additional infected dogs. In the study villages, the adult seroprevalence of infection increased from $24 \%$ in households with no infected dogs or cats to $48 \%$ in households with one to two infected dogs or cats and to $64 \%$ with three to five infected dogs or cats (25). The predicted human and bug prevalence rates of $T$. cruzi are both approximately consistent with earlier field data from 1982 and 1984, before the first professional insecticide spraying (9).

To investigate the sensitivity of model predictions to changes in key parameters, Figs. 1 and 2 were recalculated after making three separate changes, one at a time: (i) the maximum number of fourth- and fifth-instar nymphs and adult bugs that the physical infrastructure of the house will support, given an unlimited food supply, was reduced to 150 from the baseline value of 500; (ii) the number of feeding contacts per bug per spring and summer combined was increased to 10 instead of the baseline value of 5; and (iii) the relative preference of bugs for chickens was increased to 6 while their preference for dogs remained unchanged at the baseline value of 3. The predictions of the model were robust to these changes. The first few infected dogs in the household resulted in a large increase in the number of infected summer bugs, the number of infective feeding contacts per human, and the human prevalence rate. With 10 feeding contacts, keeping chickens in bedroom areas in spring reduced the bug and human prevalence rates even less than when the number of feeding contacts was 5 . Reducing the maximum number of bugs from 500 to 150 reduced the human prevalence rate from 0.63 to 0.4 .

This model indicates that an increase in the domiciliary chicken population very slightly decreases the human prevalence rate but by an amount that would be undetectable in practice. This marginal benefit for an individual household is accompanied by an increase in the size of the infected summer bug population. Because the bugs are most active and most likely to disperse to other houses as temperatures rise (26), the very slight reduction in the household prevalence rate with more chickens may be outweighed by greater risk of spreading both bug infestation and infection with $T$. cruzi to the rest of the village. These results too are robust with respect to plausible variations of the underlying assumptions of the model.

Keeping domestic animals in bedroom areas entails health and economic hazards, independent of the effect on T. cruzi infection. Domestic animals may attract or harbor other potentially dangerous vectors (such as mosquitoes, sandflies, ticks, and lice) and are associated with other pathogens infectious to humans (such as influenza, Toxocara spp., and Echinococcus spp.). Chickens repeatedly bled by bugs may be less valuable food for householders. Zooprophylactic measures may fail if vectors shift hosts (27).

The model produces a straightforward and clear result from a complex system. Keeping dogs and other highly infectious vertebrates out of bedroom areas can effectively reduce the bug and human prevalence rate, according to the model and field data. Human behavior strongly influences the transmission of $T$. cruzi infection, in addition to chemical and environmental factors that are more commonly emphasized. Low-cost, locally practicable environmental management strategies with intermittent use of insecticides can control human transmission of $T$. cruzi sustainably in rural Argentina and probably elsewhere (28). Historically, multiple simultaneous interventions that included environmental management measures proved successful in controlling malaria (29) and other vector-borne tropical diseases (30). Community education and continuous surveillance through a local health post are key requirements for effective use of the control strategies identified here.

The task force on applied research on Chagas disease (31) sponsored by the United Nations Development Program, World Health Organization, and World Bank recommends increased efforts to control triatomine vectors that occupy domestic and other habitats in the Andes and Central America. The household model described here could be extended to a spatially explicit form to take account of interactions among households and surrounding forests. A similar modeling approach could prove useful in evaluating zooprophylaxis of other infectious diseases such as malaria, Japanese encephalitis virus, visceral leishmaniasis in Brazil, and the red grouse-hare-louping ill virus system.

\section{References and Notes}

1. A. Moncayo, World Health Stat. Q. 50, 195 (1997).

2. G. A. Schmuñis, F. Zicker, A. Moncayo, Lancet 348, 1171 (1996).

3. E. L. Segura et al., Am. J. Trop. Med. Hyg. 62, 353 (2000).

4. D. M. Minter, in American Trypanosomiasis Research, Pan American Health Organization Sci. Publ. 318 (PAHO, Washington, DC, 1976) , pp. 330-337.

5. M. W. Service, Rev. Saúde Pública 25, 165 (1991).

6. G. Da Silva, Rev. Saúde Pública 3, 23 (1969).

7. J. E. Rabinovich, in Chagas' Disease Vectors, R. R Brenner, A. de la Merced Stoka, Eds. (CRC Press, Boca Raton, 1987), vol. 1, pp. 119-146.

8. J. E. Rabinovich, P. H. Himschoot, Ecol. Model. 52, 249 (1990).

9. J. E. Rabinovich, C. Wisnivesky-Colli, N. D. Solarz, R. E. Gürtler, Bull. W.H.O. 68, 737 (1990).

10. S. N. Busenberg, C. Vargas, in Mathematical Population Dynamics, Proceedings of the Second International Conference, Rutgers University, New Brunswick, NJ, 17 to 20 May 1989, O. Arino, D. E. Axelrod M. Kimmel, Eds. (Dekker, New York, 1991), pp. 283 295.

11. M. Canals, P. E. Cattan, Rev. Méd. Chile 120, 1359 (1992).

12. P. H. Himschoot, thesis, University of Buenos Aires (1993).

13. J. X. Velasco-Hernández, Theor. Popul. Biol. 46, 1 (1994).

14. M. B. Castañera, thesis, University of Buenos Aires (1999).

15. Web table 1 and supplemental text are available at Science Online at www.sciencemag.org/cgi/content/ full/293/5530/694/DC1.

16. R. E. Gürtler, M. C. Cécere, D. P. Vazquez, R. Chuit, J. E. Cohen, J. Med. Entomol. 33, 15 (1996).

17. S. Catalá, Med. Vet. Entomol. 5, 325 (1991).

18. M. C. Cecere, R. E. Gürtler, R. Chuit, J. E. Cohen, Med. Vet. Entomol. 11, 383 (1997).

19. M. C. Cecere, R. E. Gürtler, D. Canale, R. Chuit, J. E. Cohen, Bull. W.H.O. 76, 373 (1998).

20. R. E. Gürtler, J. E. Cohen, M. C. Cecere, R. Chuit, J. Appl. Ecol. 34, 699 (1997).

21. R. E. Gürtler et al., Trans. R. Soc. Trop. Med. Hyg. 85 , 741 (1991).

22. R. E. Gürtler et al., Trans. R. Soc. Trop. Med. Hyg. 87, 12 (1993).

23. R. E. Gürtler, J. E. Cohen, M. C. Cecere, R. Chuit, E. L. Segura, Am. J. Trop. Med. Hyg. 58, 748 (1998).

24. L. C. Giojalas, S. S. Catalá, S. N. Asin, D. E. Gorla, Trans. R. Soc. Trop. Med. Hyg. 84, 439 (1990).

25. R. E. Gürtler et al., Am. J. Trop. Med. Hyg. 59, 741 (1998).

26. M. J. Lehane, P. K. McEwen, C. J. Whitaker, C. J. Schofield, Acta Trop. 52, 27 (1992).

27. S. Hewitt, M. Kamal, N. Muhammad, M. Rowland, Med. Vet. Entomol. 8, 160 (1994).

28. C. J. Schofield, R. Briceno-Leon, N. Kolstrup, D. J. T. Webb, G. B. White, in Appropriate Technology in 


\section{R E P O R T S}

Vector Control, C. F. Curtis, Ed. (CRC Press, Boca Raton, FL, 1990), p. 187

29. U. Kitron, A. Spielman, Rev. Infect. Dis. 11, 391 (1989).

30. S. K. Ault, Am. J. Trop. Med. Hyg. Suppl. 50, 35 (1994).

31. Special Programme for Research and Training in Tropical Diseases, Work plan of the task force on applied research on Chagas disease (World Health
Organization, Geneva, 1999). Available at: www. who.int/tdr/grants/workplans/chagas4.htm.

32. We thank A. E. Cohen, C. Dye, J. E. Rabinovich, C. J. Schofield, and B. H. Singer for helpful comments on a previous draft. We also thank the Rockefeller Foundation for grant RF 91080, Allocation 133. J.E.C. thanks the NSF for grants BSR 9207293 and DEB 9981552, and Mr. and Mrs. W. T. Golden for hospitality during this work. R.E.G. thanks the University of
Buenos Aires, the Fulbright and Thalmann programs, and CONICET of Argentina for grants. The authors thank D. M. Canale, M. B. Castañera, I. Castro, M. C. Cecere, R. Chuit, A. Hurvitz, M. A. Lauricella, E. L. Segura, and D. P. Vázquez for sustained long-term commitment which made possible the empirical studies underlying the model.

12 March 2001; accepted 5 June 2001 
Cohen

Web Supplement

MS \#1060638

In endemic areas, $T$. cruzi infection is transmitted on three distinct spatial scales: regionally through human rural-to-urban migration and visitation between villages $(1,2)$; within a village when domestic triatomine bugs disperse among households or from surrounding sites or forests to houses, or when dogs, cats and humans visit among houses (3); and within each household, through intimate contact among humans, dogs, cats, and bugs (4). Transmission is most intense within the household. Hence, we developed a model of the seasonal transmission of T. cruzi in a single household and focussed on the means of intervention available to householders.

This Web supplement specifies the assumptions, variables, and formal structure of the model. It also lists the computing code used to generate and plot the numerical predictions of the model.

\section{Biological setting of the model}

The model aims to represent transmission of $T$. cruzi in the domestic or domiciliary area of a typical individual household in the Grand Chaco of northwestern Argentina. This area includes human sleeping quarters and the veranda covered by the same roof, as well as the occupants at night. It excludes the peridomestic area (corrals and buildings not connected to human sleeping quarters) because peridomestic $T$. infestans exhibit marginal rates of contact with domiciliary hosts and marginal $T$. cruzi infection rates $(5,6,7)$. T. infestans does not have sylvatic foci in most of its present distribution.

The model treats an individual household as a closed system. The model neglects the introduction of $T$. cruzi into the household and its spread outward from the household. The model assumes that the spatial distribution of people, dogs, chickens and bugs within the household is unchanged from the situations in which the descriptive data were gathered. Changed spatial 
arrangements could affect feeding contacts and transmission.

The model traces the household through two warm seasons within a single year, from the onset of spring to the end of summer, assuming that transmission is negligible during the cold seasons of fall and winter.

Counts of bugs refer exclusively to fourth- and fifth-instar nymphs and adult bugs. Late instars at the end of summer pass through the winter with greatly reduced activity; recommence feeding, molting, and egg-laying in spring; and die before summer. Most of the spring-born bugs disappear before the next spring. Hence, the model assumes that all bugs live for two seasons only, spring and the following summer, or summer and (after overwintering) the following spring.

Bugs acquire infection by feeding on infected humans and infected dogs $(5,6,8)$. In the spring but not the summer, chickens belong to the pool of blood meal sources for the bugs, reducing the probability of a bug's acquiring infection in the spring and increasing the bug population size in the summer.

A feeding contact (or feeding) is defined as a full blood meal on a given individual host obtained by a bug on one feeding trip or bout, regardless of the total number of bites delivered on this host to secure the blood meal. "Full" is here used to match the experimental estimation of the probability of a bug becoming infected in a single blood meal (8). Full blood meals imply an increased chance of a bug defecating on the host's skin and of potential parasite transmission.

When the continuous variables used to measure the sizes of host or bug populations include fractions, the fractions may be interpreted as the average fraction of time individuals are present in the household (e.g., 0.5 dog means a dog inside the household half time). Cats are much less preferred by $T$. infestans than humans, dogs and chickens $(5,9)$ and are omitted from the model.

\section{Assumptions of the model}

The input and output variables of the model are listed, defined and illustrated in table 1 . The user 
of the model specifies the number of humans by age groups as well as numbers of chickens, infected dogs and uninfected dogs. These numbers are assumed fixed for any run of the model. Here the modeled household contains one human aged 0 to 4 , one aged 5 to 9 , one aged 10 to 14 , one aged 25 to 29, and one aged 30 to 34 (three children, two parents). The base case assumes two chickens, two infected dogs, and no uninfected dogs. Other household compositions can be specified.

Chickens are assumed to nest or brood in bedroom areas in the spring but not the summer. The number is given by the variable $\mathrm{C}$, for "chickens." Humans, dogs and bugs are assumed to live in bedroom areas in spring and summer. The user specifies how many of the household dogs are infected with T. cruzi as the input variable DI (for "dogs infected"). All dogs are assumed infected with $T$. cruzi in these calculations, as is nearly true in the highly infested houses $(6)$, but the model can take account of any number of uninfected dogs as well (DU). The total number of dogs is D (for "dogs").

The number of bugs is treated as constant over time within each warm season (spring or summer) but differs between seasons. The suffixes $g$ and $r$ denote spring and summer, respectively. The size $\mathrm{Bg}$ of the spring bug population is a Monod function of the number of vertebrate hosts $\mathrm{Vr}=\mathrm{H}+\mathrm{R} * \mathrm{D}$ available the previous summer, where $\mathrm{R}$ is the user-specified relative feeding index of dogs and chickens as sources of feeding contacts compared to humans. With $\mathrm{R}=3$, one dog or chicken counts as three humans as far as the bugs are concerned, so a household with one person and one dog supports as many bugs as a household with four people only. The number Br of bugs in summer is a Monod function of the number of vertebrate hosts $\mathrm{Vg}=\mathrm{H}+\mathrm{R} *(\mathrm{C}+\mathrm{D})$ available in the previous spring.

The Monod function (see $\mathrm{Bg}, \mathrm{Br}$ in table 1) is consistent with experimental evidence (10) and field studies in experimental huts (11) and rural houses (12). The Monod function has two user-specified coefficients, Blimit and Vhalf. The maximum number of bugs the physical 
infrastructure of the house will support, Blimit, given an unlimited food supply, is higher if the mud walls of the house are cracked, providing places for bugs to hide and lay eggs, and is lower if the walls are smoothly plastered, if the roof of the house is built of materials resistant to bug infestation or if the roofs and walls are treated with non-professional aerosol insecticides or smokes. The value Blimit $=500$ (late-instar and adult bugs) exceeds a census of 126 late instars of T. infestans in bedrooms of a house demolished in Córdoba, Argentina (13), as well as two of the three counts of 177, 682, and 137 late instars in three houses demolished in Goiás, Brazil (14), and is approximately consistent with an estimate of the bug carrying capacity of mud and thatch houses (15). The coefficient Vhalf (for "vertebrates halfway") is the number of vertebrate blood sources (in human equivalents) sufficient to support Blimit/2 bugs.

The user also specifies five parameters of transmission of infection.

1) The number of feeding contacts per bug per spring and summer combined is $\mathrm{M}$, for "meals." With $\mathrm{M}=5$ feeding contacts per late instar and adult bug per spring and summer combined, most bugs are expected to feed on two or more vertebrate species (for example, humans and dogs). The fractions of feeding contacts from humans, dogs and chickens calculated in the model may correspond only approximately to the fractions of bugs whose guts have blood meals from each of the sources $(5,16)$, because a bug may feed multiple times on a given vertebrate host individual or species, and this repeat feeding is not detectable by routine immunological tests. For the same reason, neither the fraction of bugs fed nor the fraction of feeding contacts on a given species of vertebrate host can be interpreted as a surrogate for the number of host individuals of that species in a household.

2) The fraction $\alpha$ of a bug's $M$ annual feeding contacts that a bug takes in the season in which it is in instar 4 or 5 or adult reflects the prolonged life cycle of bugs, as estimated in experimental huts under natural climatic conditions (11). The results of the model are insensitive to the value of $\alpha$ in the range from 0.5 to 1.0 , so we took $\alpha=0.75$. The model assumes that $\mathrm{M}$ 
and $\alpha$ are independent of the number of vertebrate blood sources.

3) The bug-to-human transmission probability $\left(t_{\mathrm{B} \rightarrow \mathrm{H}}\right)$ is the probability that, in one feeding contact by one infected bug on an initially uninfected human, the human acquires infection. In all simulations reported here, $\mathrm{t}_{\mathrm{B} \rightarrow \mathrm{H}}$ is assigned the value $\mathrm{t}_{\mathrm{B} \rightarrow \mathrm{H}}=0.0008$. This value is based on numerical experimentation with possible alternatives. This value is close to the 0.0009 estimated from one field study (17) but is smaller than 0.01 assumed in a theoretical model (18).

4) The human-to-bug transmission probability $\mathrm{t}_{\mathrm{H} \rightarrow \mathrm{B}}$ (here 0.03 ) is the probability that, in one feeding by an initially uninfected bug on a seropositive human, the bug acquires infection.

5) The dog-to-bug transmission probability $t_{\mathrm{D} \rightarrow \mathrm{B}}$ (here 0.49 ) is analogous to the human-tobug transmission probability. The probabilities 0.03 and 0.49 of infecting an initially uninfected bug from a single full feeding on a $T$. cruzi-seropositive person or seropositive dog, respectively, were estimated experimentally by xenodiagnosis: laboratory-reared uninfected bugs were fed separately on seropositive people and seropositive dogs (8).

The user gives the model an arbitrary initial value of the household's prevalence among humans $(\mathrm{pH})$ at the onset of spring $(\mathrm{pH} 0)$. The model assumes that initially some humans or dogs are infected, i.e., $\mathrm{pH} 0+\mathrm{DI}>0$. Under this assumption, the value assumed for $\mathrm{pH} 0$ has no effect on the computed equilibrial human prevalence.

Successive lines of code in "model 5" below compute as follows.

1. The total number D of dogs is DI + DU.

2. The total number $\mathrm{H}$ of humans is the sum of the number of humans of each age, that is, the sum of the input vector Ha, which in these calculations is always $[1,1,1,0,0,1,1]$.

3. The number $\mathrm{Vg}$ of spring vertebrates, in human equivalents, is $H+R *(D+C)$, where $C$ is the total number of chickens.

4. The number $\mathrm{Vr}$ of summer vertebrates, in human equivalents, is $\mathrm{H}+\mathrm{R}^{*} \mathrm{D}$.

5. The number Bg of spring bugs is a Monod function of the number of previous summer 
vertebrates, Blimit*Vr/(Vhalf $+\mathrm{Vr})$.

6. The number $\mathrm{Br}$ of summer bugs is a Monod function of the number of previous spring vertebrates, Blimit*Vg/(Vhalf $+\mathrm{Vg})$.

7. The number of infected humans is $\mathrm{NH}=\mathrm{H}^{*} \mathrm{pH} 0$.

8. The probability that, in one full feeding contact, an initially uninfected bug acquires $T$. cruzi infection is $\mathrm{Tg}=(0.03 * \mathrm{NH}+0.49 * \mathrm{R} * \mathrm{DI}) / \mathrm{Vg}$ in the spring and $\mathrm{Tr}=(0.03 * \mathrm{NH}+0.49 * \mathrm{R} * \mathrm{DI}) / \mathrm{Vr}$ in the summer. The latter denominator omits chickens because they are not available to bugs in the summer. The model assumes that each bug selects the host for its next feeding contact at random among the available vertebrate blood sources (after allowing for their different host preferences). Data which have been offered as relevant to this assumption have varying interpretations (19).

9. The prevalence rate $\mathrm{pBg}$ of $T$. cruzi infection in spring bugs is $1-\left((1-\mathrm{Tg})^{\wedge}\left(\mathrm{M}^{*} \alpha\right)\right)^{*}((1-$ $\left.\operatorname{Tr})^{\wedge}\left(\mathbf{M}^{*}(1-\alpha)\right)\right)$, assuming that the bug has $\mathbf{M}^{*} \alpha$ feeding contacts in spring and $\mathbf{M}^{*}(1-\alpha)$ feeding contacts in the previous summer and that the transmission of infection is independent among feeding contacts. The prevalence rate $\mathrm{pBr}$ of $T$. cruzi infection in summer bugs is computed in the identical way, with spring and summer exchanged.

10. The spring number of infected bugs $\mathrm{NBg}$ is the product $\mathrm{Bg}^{*} \mathrm{pBg}$ of the number of spring bugs times the prevalence rate in spring bugs. The summer number of infected bugs NBr is likewise $\mathrm{Br} * \mathrm{pBr}$.

11. The average number of feeding contacts per person per year (spring and summer combined) Bitespy is the sum of the average number of feeding contacts per person in spring plus the average number of feeding contacts per person in summer. Each season-specific average number of feeding contacts per person is the quotient of the number of feeding contacts from all bugs in that season divided by the number of all vertebrate hosts in that season. For example, the spring average number of feeding contacts per person is $\left(\mathrm{Bg}^{*} \alpha+\mathrm{Br}^{*}(1-\alpha)\right) / \mathrm{Vg}$ because spring bugs 
deliver $\mathrm{Bg}^{*} \alpha$ feeding contacts to the household in spring, whereas summer bugs deliver $\mathrm{Br}^{*}(1-$ $\alpha$ ) feeding contacts to the household in spring. These feeding contacts are uniformly distributed among the human-equivalent vertebrate hosts, and each human counts as one human-equivalent. 12. The average number of feeding contacts by $T$. cruzi-infected bugs per person per year Infbitespy is the sum of the average number of feeding contacts from infected bugs per person in spring plus the average number of feeding contacts from infected bugs per person in summer. The formulas are the same as for Bitespy except that the numbers of bugs, $\mathrm{Bg}$ and $\mathrm{Br}$, are replaced by the numbers of infected bugs, $\mathrm{NBg}$ and $\mathrm{NBr}$ respectively.

13. The average number $\mathrm{Ca}$ of feeding contacts with an infected bug a human of age a has experienced over his or her lifetime thus far is the product of the person's age times the average number of feeding contacts with infected bugs per person per year, a*infbitespy. In these calculations, the input vector $\mathrm{a}=[2.5,7.5,12.5,17.5,22.5,27.5,32.5]$.

14. The prevalence rate $\mathrm{pHa}$ in humans of age a is the complement of the probability that none of the feeding contacts with an infected bug has transmitted infection to the human, that is, $\mathrm{pHa}=1$ $\left(1-\mathrm{t}_{\mathrm{B} \rightarrow \mathrm{H}}\right)^{\mathrm{Ca}}$.

15. The number $\mathrm{NHa}$ of infected humans of age a is $\mathrm{Ha}^{*} \mathrm{pHa}$, the product of the number of humans of age a times the prevalence rate in humans of age a.

16. The average (over all ages) of the prevalence rate of infection in humans is the sum of the number NHa of infected humans of all ages, divided by the total number of humans of all ages. This quantity is $\mathrm{pH} 1$, the net result of the operation of the model through the spring and summer seasons starting from the initial prevalence rate $\mathrm{pH} 0$ at the onset of spring.

17. The fraction of feeding contacts with humans, dogs and chickens in spring and summer are calculated, using similar reasoning, in the concluding lines of model5.

Additional assumptions of the model are:

1) Transmission processes and probabilities are the same in spring and summer. The only 
difference is that chickens are present in bedroom areas in spring and absent in summer.

2) T. cruzi infection does not affect significantly the vital parameters (feeding, growth, survival, and reproduction) of hosts and bugs.

3) Susceptibility to T. cruzi infection is independent of bug stage (for fourth- and fifthinstar nymphs and adult bugs), age and sex of mammal hosts, and ambient temperature.

4) The probability of bug infection after a single full blood meal on an infected host is independent of host age and ambient temperature.

5) Transmission mediated by T. infestans is the sole route of infection. Vertical transmission of $T$. cruzi from an infected human mother to her infant and transmission by transfusion are ignored.

6) The probability of transmission to an uninfected human per feeding contact is independent of (a) the intensity of bug infection [number of trypomastigote parasites (infectious forms of T. cruzi) per bug], (b) bug stage, (c) previous bloodmeal sources, (d) absolute or relative (per host) density of bugs, and (e) ambient temperature.

7) Host bloodmeal sources do not affect significantly the bugs' vital parameters, bloodmeal size, and acquisition or development of T. cruzi infection.

8) Individuals within each species of hosts, bug and parasite do not differ significantly in their course of infection.

9) The population dynamics of hosts, bugs and parasites are not significant for the equilibrium human prevalence of infection with $T$. cruzi, that is, it is sufficient to consider the effect of the steady-state population sizes on human prevalence.

Of these assumptions, 1, 2, and 7 have supporting evidence or are a reasonable approximation given current knowledge. Only partial evidence is available regarding assumption 6(d). A multiple linear regression (20) showed that, when T. infestans was interrupted while it was biting, the time to the first fecal drop was inversely and significantly related to blood intake 
and was directly and significantly related to how long the bug was starved before feeding and how much the bug weighed at the start of feeding. Although previous experiments using restrained mice or unrestrained chickens held in small boxes showed that T. infestans blood intake was negatively density dependent, it does not necessarily follow that a feeding contact with a single bug at high bug population density has a lower risk of transmitting infection to an uninfected mammalian host than a feeding contact with a single bug at a low bug population density. The relation between bug population density and the probability of transmission by a single feeding contact with an infected bug remains to be assessed empirically. Mice have been infected with as few as $25 T$. cruzi trypomastigotes inoculated intraperitoneally (21), whereas field-collected $T$. infestans had millions of infective trypomastigotes per milliliter of bug feces (22). Assumptions 3, 4, 5, and 8 are simplifications of convenience, against which there is some contradictory evidence. For example, contrary to assumption 4 , children have many more trypomastigotes in their blood than older people with chronic infections and are therefore more infectious than adults (8). Dogs also may be slightly more infectious in the early than in the late chronic phase (8). Assumption 9 is appropriate for an equilibrium model applied to a chronic endemic disease transmitted by a Kstrategist insect vector living close to equilibrium abundance $(3,10)$.

The prevalence rates neglect the latency between entry of $T$. cruzi into a susceptible host and that host's becoming infectious, because latent periods of bugs and hosts (1 to 3 weeks) are negligible compared to the remaining average lifetime of a dog, human or bug at the time when each acquires the infection.

\section{Analysis of the model}

The steady-state condition $\mathrm{pH} 0=\mathrm{pH} 1$ means that the human prevalence rate at the end of summer equals the human prevalence rate at the onset of spring. This condition has exactly one mathematical solution, so the output of the model is uniquely defined, apart from limitations of numerical analysis in finding the mathematical solution. This steady-state solution $\mathrm{pH}$ is computed 
approximately by numerical iteration (repeatedly replacing $\mathrm{pH} 0$ with $\mathrm{pH} 1$ ) by the Matlab function iterate 5 until the initial human prevalence rate $\mathrm{pH} 0$ at the onset of spring differs from the final human prevalence rate $\mathrm{pH} 1$ at the end of summer by no more than the user-specified criterion $\delta$ for convergence, here taken as 0.001 ; i.e., the iteration continues until $|\mathrm{pH} 0-\mathrm{pH} 1|<\delta=0.001$.

The Matlab function chidogtable5 (for "chicken-dog table") investigates how the assumed numbers of infected dogs and chickens affects the steady-state human prevalence of T. cruzi infection and other characteristics of the household system, such as bug population sizes by season. This function is the computational alternative to ethically impossible field experimentation. Fifteen numerical values $[0,0.1,0.2,0.3,0.4,0.6,0.8,1.0,1.5,2,2.5,3,4,5$, and 6] were assigned in succession to the number of chickens $C$, and, for each value of $C$, the same 15 numerical values were assigned in succession to the number of infected dogs DI. Thus, model outputs were produced for $225=15 \times 15$ combinations of the numbers of chickens and infected dogs. These values for the input variables "chickens" and "dogs" were selected to display clearly how the model behaves in the region where output variables change rapidly, rather than to reflect the actual distribution of the numbers of chickens and infected dogs per household in the villages studied. All other parameters remained constant at the values shown in table 1.

\section{Sensitivity analyses}

The printed paper reports a sensitivity analysis with respect to the parameter values of Blimit, M, and R (see table 1 for definitions). In addition, four related models that differed in detailed structure were investigated numerically. These other models differed in the absence of seasonality, and (among the seasonal models) in the seasonality of keeping chickens indoors, in the dependence of the bug population size on the number of vertebrate hosts in the present versus prior season, and in other minor respects. Although the quantitative predictions of the models varied, the major conclusions for the control of T. cruzi transmission within households were remarkably robust. 
If the model were simplified by replacing the age-structured human population with a single age group, the human prevalence rate could be significantly overestimated. Because the curve of the human prevalence rate as a function of age is concave in this model, using a single age group in which the age is the average age $\mathrm{Abar}=\operatorname{sum}(\mathrm{a} * \mathrm{Ha}) / \mathrm{H}$ will increase the predicted human prevalence rate as a mathematical consequence of Jensen's inequality. Numerical calculations not shown here indicate that ignoring age structure could introduce substantial bias.

\section{Other related work}

Two independent simulation models assessed the effects of chickens on the household transmission of $T$. cruzi. In the presence of humans and dogs infected with $T$. cruzi, maintaining 10 or 20 chickens in domiciliary areas depressed bug and human prevalence rates of infection substantially (23). The presence of a single brooding chicken in domiciliary areas in both spring and summer decreased the daily rate of potentially infective feeding contacts experienced by humans but did not significantly reduce the human incidence rate (24).

An existing model of zooprophylaxis in malaria (25) omits seasonality. A model for the insect-transmitted African horse sickness includes seasonality (26) but deals with only two susceptible host species that differ in infectivity and pathogen-induced mortality. In the red grouse-hare-louping ill virus system (27), the nonviremic hosts can amplify the tick (vector) population and cause the virus to persist or can cause the infection to die out. None of these models made any comparison with field data.

\section{Computing software}

All numerical calculations reported here were carried out on a Dell personal computer using MATLAB 5.2 (28). The three Matlab functions used to implement the model, namely, chidogtable5, iterate5, model5, are listed below. The user first defines numerical values for the variables a, Ha, C, DI, DU, R, Blimit, Vhalf, M, t, pHo, alpha, delta, chickens, dogs, which are defined in the internal comments of chidogtable5, then 
commands:

$$
\text { out=chidogtable5 (a, Ha, C, DI , DU, R, Blimit, Vhalf , M, t, pH0, alpha, }
$$

delta, chickens, dogs);

The function chidogtable5 invokes the function iterate5, which in turn invokes model5. The function iterate 5 determines a steady-state human prevalence rate by iteration of model5. The function chidogtable5 computes the steady-state human prevalence rates associated with different combinations of numbers of chickens and dogs, and plots the results.

\section{Function model5}

function

[pH1 , NHa , Bg , Br , pBg , pBr , NBg , NBr , bitespy , infbitespy, humbloodg, do gbloodg, chibloodg, humbloodr, dogbloodr , chibloodr ] =model5 ( a , Ha , C ,DI , DU, R, Blimit, Vhalf, M, t, pHo, alpha)

․ pH1 , NHa , Bg , Br , pBg , pBr , NBg , NBr , bi tespy, infbitespy, humbloodg, dogb loodg, chibloodg, humbloodr, dogbloodr, chibloodr] =model5 ( $\mathrm{a}$, Ha , C, D I , DU, R, Blimit, Vhalf , M, t, pHo, alpha)

\%Model of Chagas transmission in a household: 2 seasons (spring, summer)

\%Bug population size is set in previous season

\%Bug prob. of acquiring infection and bug prevalence rate are set in season of adulthood

\%uffix $g=$ spring, $r=$ summer

\%a =vector of midpoints of age groups

$\% \mathrm{Ha}=$ vector of number of people in each age group in household

$\div \mathrm{C}=$ chickens

$\because \mathrm{DI}=$ dogs infected

$\% \mathrm{DU}=$ dogs uninfected

$\% R=$ relative attractiveness of dogs and chickens as blood meals wrt humans 
\%Blimit=maximum number of bugs given unrestricted food

\%Vhalf=no.of vertebrate blood sources (in human equivalents)

sufficient to support Blimit/2 bugs

$\% \mathrm{M}=$ meals per bug per summer

$\%$ t =prob. that, in one bite by one infected bug on initially uninfected human, human acquires infection

$\circ \mathrm{pHO}=$ initial prevalence rate of Chagas infection in humans

\%alpha = fraction of blood meals that adult bugs take during season of adulthood

27 December 1999; 9, 14, 25, 29 March 2000

$\mathrm{D}=\mathrm{DI}+\mathrm{DU} ;$

$\mathrm{H}=\operatorname{sum}(\mathrm{Ha}) ;$

$\mathrm{Vg}=\mathrm{H}+\mathrm{R} *(\mathrm{D}+\mathrm{C}) ; \quad$ \%spring vertebrate blood sources, in human equivalents, chickens indoors

$\mathrm{Vr}=\mathrm{H}+\mathrm{R} * \mathrm{D} ; \quad$ \%summer vertebrate blood sources, in human equivalents, chickens outdoors

$\mathrm{Bg}=\mathrm{Blimit} * \mathrm{Vr} /(\mathrm{Vhal} f+\mathrm{Vr})$; ospring IV-V-adult bugs in household set by summer vertebrates

$\mathrm{Br}=\mathrm{Blimit} * \mathrm{Vg} /(\mathrm{Vhal} f+\mathrm{Vg})$; $\%$ summer IV-V-adult bugs in household set by spring vertebrates

$\mathrm{NH}=\mathrm{H} * \mathrm{pHO} ; \quad$ oinitial prevalence in humans

$\mathrm{Tg}=(0.03 * \mathrm{NH}+0.49 * \mathrm{R} * \mathrm{DI}) / \mathrm{Vg} ; \quad$ \%spring prob. that an initially

uninfected bug acquires infection in one blood meal

$\operatorname{Tr}=(0.03 * \mathrm{NH}+0.49 * \mathrm{R} * \mathrm{DI}) / \mathrm{Vr} ; \quad$ \%summer prob. that an initially

uninfected bug acquires infection in one blood meal

$\mathrm{pBg}=1-\left((1-\mathrm{Tg})^{\wedge}(\mathrm{M} * \mathrm{alpha})\right) *\left((1-\mathrm{Tr})^{\wedge}(\mathrm{M} *(1-\mathrm{alpha}))\right) ; \quad$ \%spring

prevalence rate in spring-late-instar and adult bugs $\operatorname{pBr}=1-\left((1-\mathrm{Tr})^{\wedge}(\mathrm{M} * \mathrm{alpha})\right) *\left((1-\mathrm{Tg})^{\wedge}(\mathrm{M} *(1-\mathrm{alpha}))\right) ; \quad$ \%summer

prevalence rate in summer-late-instar and adult bugs 


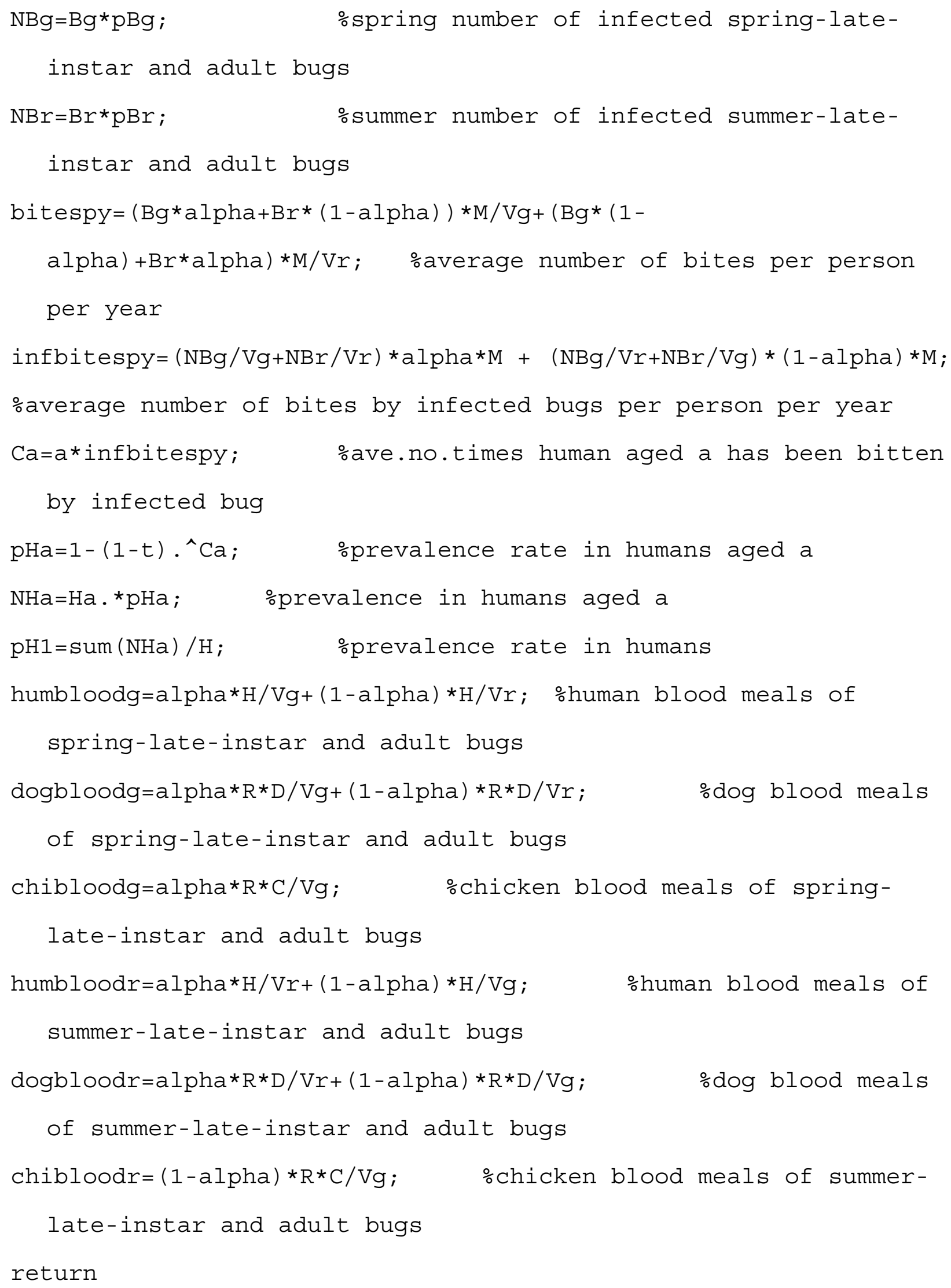




\section{Function iterate5}

function

[pH1 , $\mathrm{PHa}, \mathrm{Bg}, \mathrm{Br}, \mathrm{pBg}, \mathrm{pBr}$, NBg , NBr , bitespy , infbitespy, humbloodg, do gbloodg, chibloodg, humbloodr, dogbloodr, chibloodr, aveage, aveinfa ge ] =iterate5 ( $a, H a, C, D I, D U, R, B l i m i t, V h a l f, M, t, p H 0, a l p h a$, delta) ․ $[\mathrm{pH} 1$, $\mathrm{PHa}$, $\mathrm{Bg}, \mathrm{Br}, \mathrm{pBg}, \mathrm{pBr}, \mathrm{NBg}, \mathrm{NBr}$, bitespy , infbitespy , humbloodg , dogb loodg, chibloodg, humbloodr, dogbloodr, chibloodr] =iterates (a , Ha , C ,DI , DU, R, Blimit, Vhalf , M, t, pH0, alpha, delta)

\%27 December 1999, rev. 2 Jan 2000, 9, 14, 25 March 2000 $\mathrm{pH} 1=\mathrm{pHO} ;$

pH2 =model $5(\mathrm{a}, \mathrm{Ha}, \mathrm{C}, \mathrm{DI}, \mathrm{DU}, \mathrm{R}, \mathrm{Blimit}, \mathrm{Vhalf}, \mathrm{M}, \mathrm{t}, \mathrm{pH}$, alpha $)$;

while abs (pH2-pH1) >delta

$\mathrm{pH} 1=\mathrm{pH} 2$ ；

pH2 =model5 ( $a, H a, C, D I, D U, R, B l i m i t$, Vhalf $, \mathrm{M}, t, \mathrm{pH} 1, \mathrm{alpha})$;

end

[pH1 , PHa , Bg , Br , pBg , pBr , NBg , NBr , bitespy , infbitespy, humbloodg, dogbl oodg, chibloodg, humbloodr, dogbloodr, chibloodr] =model5 (a , Ha , C, DI ,DU, R, Blimit, Vhalf , M, t, pH0, alpha) ;

aveage $=\operatorname{sum}(\mathrm{a} . * \mathrm{Ha}) / \operatorname{sum}(\mathrm{Ha}) ; \quad$ \%average age of humans aveinfage $=\operatorname{sum}(a . * \mathrm{PHa}) / \operatorname{sum}(\mathrm{PHa}) ;$ oaverage age of infected humans return

\section{Function chidogtable5}

function

out =chidogtable5 (a, Ha, C, DI , DU, R, Blimit, Vhalf , M, t, pH0, alpha, del ta, chickens, dogs)

\%out=chidogtable5 ( $\mathrm{a}, \mathrm{Ha}, \mathrm{C}, \mathrm{DI}, \mathrm{DU}, \mathrm{R}, \mathrm{Blimit}, \mathrm{Vhalf}, \mathrm{M}, \mathrm{t}, \mathrm{pH}$, alpha, delta , chickens, dogs) 
\%Model of Chagas transmission in a household: 2 seasons (spring, summer)

\%Bug population size is set in previous season

\%Suffix $g=$ spring, $r=$ summer

$\%$ a =vector of midpoints of age groups

$\% H a=v e c t o r$ of number of people in each age group in household

$\because \mathrm{C}=$ chickens

$\% \mathrm{DI}=$ dogs infected

$\% \mathrm{DU}=$ dogs uninfected

$\% R$ =relative attractiveness of dogs and chickens as feeding contacts wrt humans

\%Blimit=maximum number of bugs given unrestricted food

\%Vhalf=no. of vertebrate blood sources (in human equivalents)

sufficient to support Blimit/2 bugs

$\because \mathrm{M}=$ feeding contacts per bug per spring and summer combined

$\%$ t =prob. that, in one bite by one infected bug on initially uninfected human, human acquires infection

$\%$ pHO=initial prevalence rate of Chagas infection in humans

\%alpha $=$ fraction of feeding contacts that late-instar and adult

bugs take during season when they become late-instar or adult

\%delta=threshold for quasi-convergence of iteration: steady-state

iff $|\mathrm{pH} 2-\mathrm{pH} 1|<\operatorname{delta}$

\%chickens=vector of numbers of chickens to assume in household

\%dogs=vector of numbers of dogs to assume in household

\%out level 1 number of chickens in household

\% level 2 number of dogs in household

\% level $3 \mathrm{pH}$ prevalence rate in humans

\% level 4 Bg late-instar and adult bugs in household in spring

\% level 5 Br late-instar and adult bugs in household in summer 
\% level 6 pBg prevalence rate in bugs in spring

\% level 7 pBr prevalence rate in bugs in summer

\% level $8 \mathrm{NBg}$ number of infected bugs in spring

\% level $9 \mathrm{NBr}$ number of infected bugs in summer

\% level 10 bitespy average number of bites per person per year

\% level 11 infbitespy average number of bites by infected bugs per person per year

\% level 12 humbloodg fraction of feeding contacts on human blood in spring

\% level 13 dogbloodg fraction of feeding contacts on dog blood in spring

\% level 14 chibloodg fraction of feeding contacts on chicken blood in spring

\% level 15 aveage average age of humans

\% level 16 aveinfage average age of infected humans

\% level 17 humbloodr fraction of feeding contacts on human blood in summer

\% level 18 dogbloodr fraction of feeding contacts on dog blood in summer

\% level 19 chibloodr fraction of feeding contacts on chicken blood in summer

\%29 December 1999, rev. 2 January 2000, 25 March 2000, 7 May $2000 ; 23$ May 2000; 6 May 2001

fnt=22; $\quad$ ofont size for lettering of figures

fntic=18; \%font size for labeling of tic marks lc=length (chickens) ;

ld=length (dogs);

[chigrid, doggrid]=meshgrid (chickens, dogs); \%grid for each combination of chickens \& dogs 


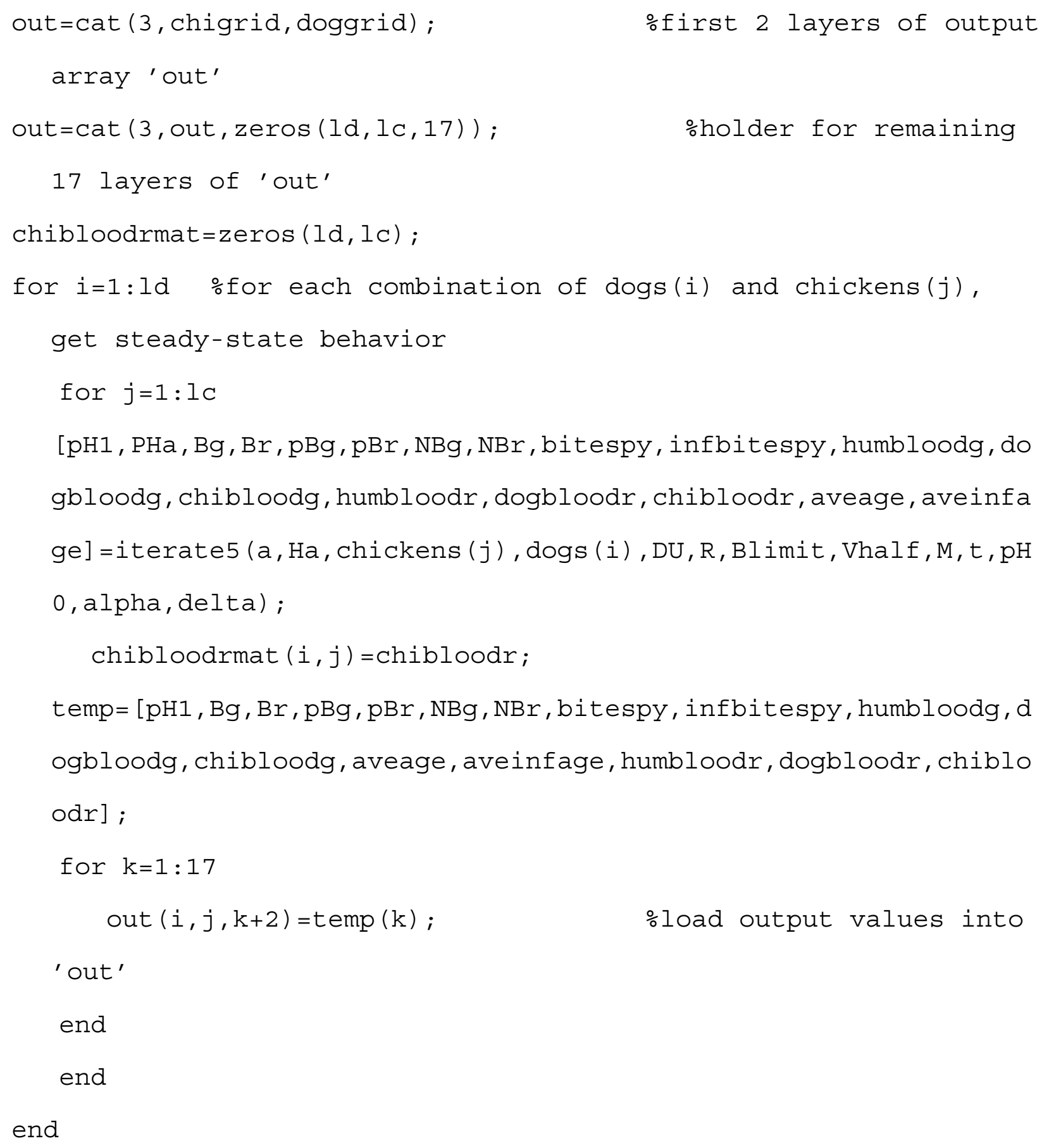


xlabel ('chicken feedings','Fontsize', fnt), ylabel('dog feedings','FontSize', fnt), zlabel ('bug prevalence rate','Fontsize', fnt)

print -deps -tiff bloodmealssummerbugprevrate.eps

\%Figure 1D, humchibloodmealsvisummerbugs

figure (16)

$\mathrm{x}=[\operatorname{out}(:,:, 5)$, out $(:,:, 5)] ; \quad \%$ late-instar and adult bugs in household in SUMMER ONLY!!!!

$\mathrm{y} 1=[\operatorname{out}(:,:, 12)$, out $(:,:, 17)] ; \%$ humbloodg and humbloodr y2=[out $(:,:, 14)$, out $(:,:, 19)] ;$ \%chibloodg and chibloodr $h=p l o t\left(x(:), y 1(:), \mathrm{O}^{\prime}, \mathrm{x}(:), \mathrm{y} 2(:), \mathrm{x}^{\prime}\right)$; set (gca, 'fontsize', fntic) title('spring \& summer feeding contacts','Fontsize', fnt) xlabel ('summer bugs in household','FontSize', fnt), ylabel ('human

(o), chicken (x) feeding contacts','FontSize', fnt)

\%print

print -deps -tiff humchibloodmealsvsummerbugs.eps

\%Figure 2A springbugsinhousehold

figure (1)

mesh (chigrid, doggrid, out $(:,:, 4))$

colormap ( [ $\left.\left.\begin{array}{lll}0 & 0 & 0\end{array}\right]\right)$

title('spring bugs in household','FontSize', fnt)

xlabel ('chickens', 'FontSize', fnt), ylabel ('dogs',' Fontsize', fnt), z label ('spring bugs','Fontsize', fnt)

set (gca,' fontsize', fntic)

oprint

print -deps -tiff springbugsinhousehold.eps 
\%Figure 2B summerbugsinhousehold

figure (2)

mesh (chigrid, doggrid, out $(:,:, 5)$ )

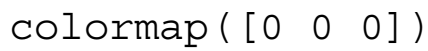

title('summer bugs in household','FontSize', fnt)

xlabel ('Chickens' ,'FontSize', fnt),ylabel ('dogs' ,'Fontsize', fnt),z

label ('summer bugs','FontSize', fnt)

set (gca,'fontsize', fntic)

oprint

print -deps -tiff summerbugsinhousehold.eps

\%Figure 2C humanbloodmeals

figure (3)

mesh (chigrid, doggrid, out (:, : , 12))

colormap $\left(\left[\begin{array}{lll}0 & 0 & 0\end{array}\right]\right)$

title('spring feeding contacts with humans','Fontsize', fnt)

xlabel ('chickens','Fontsize', fnt),ylabel ('dogs',' Fontsize', fnt), z

label('fraction of feeding contacts with

humans', 'Fontsize', fnt)

set (gca, 'fontsize', fntic)

oprint

print -deps -tiff humanbloodmeals.eps

\%Figure 2D springdogbloodmeals

figure (4)

mesh (chigrid, doggrid, out (:, : , 13))

colormap ( [ $\left.\left.\begin{array}{lll}0 & 0 & 0\end{array}\right]\right)$

title('spring feeding contacts with dogs','Fontsize', fnt) 
xlabel ('chickens' ,'FontSize', fnt),ylabel ('dogs' ,'Fontsize', fnt), z

label('fraction of feeding contacts with dogs','Fontsize',fnt)

set (gca,' fontsize', fntic)

\%print

print -deps -tiff springdogbloodmeals.eps

\%Figure $2 \mathrm{E}$ prevrateinspringbugs

figure (6)

mesh (chigrid, doggrid, out $(:,:, 6)$ )

colormap ( [ $\left.\left.\begin{array}{lll}0 & 0 & 0\end{array}\right]\right)$

title('prevalence rate in spring bugs','FontSize', fnt)

xlabel ('chickens', 'FontSize', fnt), ylabel ('dogs',' Fontsize', fnt), z

label ('prevalence rate in bugs','FontSize', fnt)

set (gca, 'fontsize', fntic)

oprint

print -deps -tiff prevrateinspringbugs.eps

\%Figure $2 \mathrm{~F}$ infectedspringbugs

figure ( 8)

mesh (chigrid, doggrid, out $(:,:, 8)$ )

colormap ( [ $\left.\left.\begin{array}{lll}0 & 0 & 0\end{array}\right]\right)$

title('infected spring bugs','FontSize', fnt)

xlabel ('chickens' ,'FontSize', fnt), ylabel ('dogs' ,'FontSize', fnt) , z

label ('number of infected bugs','Fontsize', fnt)

set (gca, 'fontsize', fntic)

。print

print -deps -tiff infectedspringbugs.eps 
figure (9)

mesh (chigrid, doggrid, out $(:,:, 9)$ )

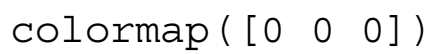

title('infected summer bugs','FontSize',fnt)

xlabel ('Chickens',' FontSize', fnt),ylabel ('dogs',' FontSize',fnt), z

label ('number of infected bugs','FontSize',fnt)

set (gca,'fontsize', fntic)

ஃprint

print -deps -tiff infectedsummerbugs.eps

\%Figure $2 \mathrm{H}$ feedingsperhumanyear

figure (10)

mesh (chigrid, doggrid, out $(:,:, 10)$ )

colormap ( $\left.\left[\begin{array}{lll}0 & 0 & 0\end{array}\right]\right)$

title('feeding contacts per human','FontSize', fnt)

xlabel ('Chickens',' FontSize', fnt),ylabel ('dogs',' FontSize',fnt),z

label ('feeding contacts per human','FontSize',fnt)

set (gca,' fontsize', fntic)

ஃprint

print -deps -tiff feedingsperhumanyear.eps

\%Figure 2I humanprevalencerate

figure (12)

mesh (chigrid, doggrid, out $(:,:, 3)$ )

colormap ( $\left.\left[\begin{array}{lll}0 & 0 & 0\end{array}\right]\right)$

title('human prevalence rate','FontSize', fnt)

xlabel ('chickens',' FontSize', fnt),ylabel ('dogs','FontSize',fnt) , z

label ('human prevalence rate','FontSize',fnt)

set (gca,' fontsize', fntic) 
\%print

print -deps -tiff humanprevalencerate.eps

return 
Web table 1. Parameters, calculated variables and descriptive statistics of the model.

\begin{tabular}{|c|c|c|}
\hline Symbol & Meaning & $\begin{array}{l}\text { Formula or illustrative value in } \\
\text { base case at equilibrium }\end{array}$ \\
\hline a & $\begin{array}{l}\text { Age groups of humans in household (vector of midpoints of ages, } \\
\text { measured in years) }\end{array}$ & $\begin{array}{l}{[2.57 .512 .517 .522 .527 .5} \\
32.5]\end{array}$ \\
\hline $\mathrm{Ha}$ & Number of humans in each age group (vector of values) & 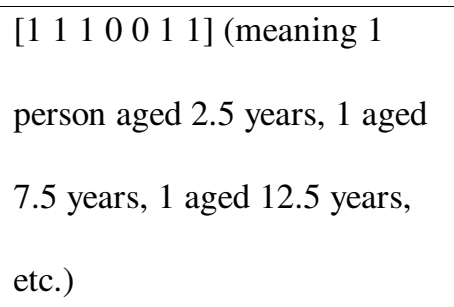 \\
\hline $\mathrm{H}$ & Number of humans in household & $\begin{array}{l}\text { sum of } \mathrm{Ha} \\
\mathrm{Ha}=5 \text { people }\end{array}$ \\
\hline DI & Number of dogs infected with $T$. cruzi & $\mathrm{DI}=2$ infected dogs \\
\hline DU & Number of dogs uninfected with $T$. cruzi & $\mathrm{DU}=0$ uninfected dogs \\
\hline $\mathrm{D}$ & Number of household dogs & $\begin{array}{l}\mathrm{DI}+\mathrm{DU} \\
\mathrm{D}=2 \mathrm{dogs}\end{array}$ \\
\hline $\mathrm{C}$ & Number of household chickens & $\mathrm{C}=2$ chickens \\
\hline $\mathrm{R}$ & $\begin{array}{l}\text { Relative feeding index of dogs and chickens as sources of feeding } \\
\text { contacts compared to humans }\end{array}$ & $\begin{array}{l}\mathrm{R}=3 \text { dog or chicken } \\
\text { feedings per human feeding }\end{array}$ \\
\hline $\mathrm{Vg}, \mathrm{Vr}$ & $\begin{array}{l}\text { Number of vertebrate blood sources, in human equivalents, in } \\
\text { spring }(\mathrm{Vg}) \text { and summer }(\mathrm{Vr})\end{array}$ & $\begin{array}{l}\mathrm{Vg}=\mathrm{H}+\mathrm{R} *(\mathrm{D}+\mathrm{C}), \mathrm{Vr}= \\
\mathrm{H}+\mathrm{R} * \mathrm{D} \\
\mathrm{Vg}=17 \text { vertebrates in } \\
\text { spring, } \\
\mathrm{Vr}=11 \text { vertebrates in } \\
\text { summer }\end{array}$ \\
\hline Blimit & $\begin{array}{l}\text { Maximum number of fourth- and fifth-instar nymphs and adult } \\
\text { bugs the physical infrastructure of the house will support, given } \\
\text { an unlimited food supply }\end{array}$ & $\begin{array}{l}\text { Blimit = } 500 \text { large and late- } \\
\text { stage bugs }\end{array}$ \\
\hline
\end{tabular}




\begin{tabular}{|c|c|c|}
\hline Vhalf & $\begin{array}{l}\text { Number of vertebrate blood sources (in human equivalents) } \\
\text { sufficient to support Blimit/2 bugs }\end{array}$ & Vhalf $=7$ vertebrates \\
\hline $\mathrm{Bg}, \mathrm{Br}$ & $\begin{array}{l}\text { Number of fourth- and fifth-instar nymphs and adult bugs in } \\
\text { spring }(\mathrm{Bg}) \text { and summer }(\mathrm{Br}) \text { that can be supported at steady- } \\
\text { state by the vertebrate blood sources in the household }\end{array}$ & $\begin{array}{l}\mathrm{Br}=\mathrm{Blimit} * \mathrm{Vg} /(\mathrm{Vg}+\text { Vhalf }) \\
\mathrm{Bg}=\mathrm{Blimit} * \mathrm{Vr} /(\mathrm{Vr}+\text { Vhalf }) \\
\mathrm{Br}=354.2 \text { bugs in summer, } \\
\mathrm{Bg}=305.6 \text { bugs in spring }\end{array}$ \\
\hline $\mathrm{M}$ & $\begin{array}{l}\text { Number of feeding contacts per fourth- or fifth-instar nymph or } \\
\text { adult bug per spring and summer combined }\end{array}$ & $\begin{array}{l}\mathrm{M}=5 \text { feeding contacts per } \\
\text { bug per summer and spring }\end{array}$ \\
\hline pH0 & $\begin{array}{l}\text { Human prevalence rate in late winter (fraction of all humans } \\
\text { infected with } T \text {. cruzi) }\end{array}$ & $\begin{array}{l}\text { initial } \mathrm{pH} 0=0.8 \\
\mathrm{pH} 0=\mathrm{pH} 1 \text { at equilibrium }\end{array}$ \\
\hline$t_{B \rightarrow H}$ & $\begin{array}{l}\text { Transmission probability to human = probability that, in one } \\
\text { feeding by one infected bug on an initially uninfected human, the } \\
\text { human acquires infection }\end{array}$ & $\mathrm{t}_{\mathrm{B} \rightarrow \mathrm{H}}=0.0008$ \\
\hline$t_{\mathrm{H} \rightarrow \mathrm{B}}$ & $\begin{array}{l}\text { Probability that, in one feeding by an initially uninfected bug on } \\
\text { an infected (seropositive) human, the bug acquires infection }\end{array}$ & $\mathrm{t}_{\mathrm{H} \rightarrow \mathrm{B}}=0.03$ \\
\hline$t_{\mathrm{D} \rightarrow \mathrm{B}}$ & $\begin{array}{l}\text { Probability that, in one feeding by an initially uninfected bug on } \\
\text { an infected (seropositive) dog, the bug acquires infection }\end{array}$ & $\mathrm{t}_{\mathrm{D} \rightarrow \mathrm{B}}=0.49$ \\
\hline $\mathrm{Tg}, \mathrm{Tr}$ & $\begin{array}{l}\text { Transmission probability to bug in spring (Tg) and summer (Tr) } \\
=\text { probability that, in one blood meal, an initially uninfected bug } \\
\text { acquires infection }\end{array}$ & $\begin{array}{l}\mathrm{Tg}=(0.03 * \mathrm{NH}+ \\
0.49 * \mathrm{R} * \mathrm{DI}) / \mathrm{Vg} \\
\mathrm{Tr}=(0.03 * \mathrm{NH}+ \\
0.49 * \mathrm{R} * \mathrm{DI}) / \mathrm{Vr}\end{array}$ \\
\hline$\alpha$ & $\begin{array}{l}\text { Fraction of feedings a bug takes in the season the bug is in instar } \\
4 \text { or } 5 \text { or adult }\end{array}$ & $\alpha=0.75$ \\
\hline $\mathrm{pBg}, \mathrm{pBr}$ & $\begin{array}{l}\text { Bug prevalence rates in spring }(\mathrm{pBg}) \text { and summer }(\mathrm{pBr}) \\
\text { (fractions of bugs infected with T. cruzi in spring and summer) }\end{array}$ & $\begin{array}{l}\mathrm{pBg}=1-\left((1-\mathrm{Tg})^{\wedge}\left(\alpha^{*} \mathrm{M}\right)\right)^{*}((1- \\
\left.\operatorname{Tr})^{\wedge}\left((1-\alpha)^{*} \mathrm{M}\right)\right) \\
\mathrm{pBr}=1-\left((1-\mathrm{Tr})^{\wedge}\left(\alpha^{*} \mathrm{M}\right)\right)^{*}((1- \\
\left.\mathrm{Tg})^{\wedge}\left((1-\alpha)^{*} \mathrm{M}\right)\right) \\
\mathrm{pBg}=0.6839 \text { in spring } \\
\mathrm{pBr}=0.7702 \text { in summer }\end{array}$ \\
\hline
\end{tabular}




\begin{tabular}{|c|c|c|}
\hline $\mathrm{NBg}, \mathrm{NBr}$ & Number of infected bugs in spring $(\mathrm{NBg})$ and summer $(\mathrm{NBr})$ & $\begin{array}{l}\mathrm{NBg}=\mathrm{Bg} * \mathrm{pBg}, \mathrm{NBr}=\mathrm{Br} * \mathrm{pBr} \\
\mathrm{NBg}=209.0 \text { infected bugs in } \\
\text { spring, } \mathrm{NBr}=272.8 \text { infected } \\
\text { bugs in summer }\end{array}$ \\
\hline Bitespy & Number of feeding contacts per human per year & $\begin{array}{l}\left(\mathrm{Bg}^{*} \alpha+\mathrm{Br} *(1-\alpha)\right)^{*} \mathrm{M} / \mathrm{Vg}+ \\
\left(\mathrm{Bg}^{*}(1-\alpha)+\mathrm{Br}^{*} \alpha\right)^{*} \mathrm{M} / \mathrm{Vr} \\
\text { Bitespy=248.9 feeding } \\
\text { contacts per human per year }\end{array}$ \\
\hline Infbitespy & $\begin{array}{l}\text { Number of potentially infective feeding contacts per human per } \\
\text { year }\end{array}$ & $\begin{array}{l}(\mathrm{NBg} / \mathrm{Vg}+\mathrm{NBr} / \mathrm{Vr})^{*} \alpha^{*} \mathrm{M}+ \\
(\mathrm{NBg} / \mathrm{Vr}+\mathrm{NBr} / \mathrm{Vg})^{*}(1-\alpha)^{*} \mathrm{M} \\
\text { Infbitespy=182.9 infective } \\
\text { feeding contacts per human } \\
\text { per year }\end{array}$ \\
\hline $\mathrm{Ca}$ & $\begin{array}{l}\text { Contacts by age a = average number of times a human in each } \\
\text { age-group a has had a feeding contact with an infected bug } \\
\text { (vector of values) }\end{array}$ & a*Infbitespy \\
\hline $\mathrm{pHa}$ & $\begin{array}{l}\text { Prevalence rate in humans in each age-group a (vector of } \\
\text { values) }\end{array}$ & $\mathrm{pHa}=1-\left(1-\mathrm{t}_{\mathrm{B} \rightarrow \mathrm{H}}\right)^{\wedge} \mathrm{Ca}$ \\
\hline Nha & $\begin{array}{l}\text { Expected number of infected humans in each age-group a } \\
\text { (vector of values) }\end{array}$ & $\mathrm{Ha}^{*} \mathrm{pHa}$ \\
\hline $\mathrm{NH}$ & Total number of infected humans & Sum of Nha \\
\hline pH1 & Human prevalence rate calculated through one cycle of the model & $\begin{array}{l}\mathrm{pH} 1=\mathrm{NH} / \mathrm{H} \\
\mathrm{pH} 1=0.7572 \text { at end of } \\
\text { summer if } \mathrm{pH} 0=0.8 \text { at onset } \\
\text { of spring }\end{array}$ \\
\hline $\begin{array}{l}\text { Humblood } \\
\text { g }\end{array}$ & $\begin{array}{l}\text { Fraction of feeding contacts from humans in bugs collected in } \\
\text { spring }\end{array}$ & $\begin{array}{l}\text { Humbloodg }=\alpha^{*} \mathrm{H} / \mathrm{Vg}+(1- \\
\alpha)^{*} \mathrm{H} / \mathrm{Vr}\end{array}$ \\
\hline
\end{tabular}




\begin{tabular}{|c|c|c|}
\hline Dogbloodg & $\begin{array}{l}\text { Fraction of feeding contacts from dogs in bugs collected in } \\
\text { spring }\end{array}$ & $\begin{array}{l}\text { Dogbloodg }=\alpha * \mathrm{R}^{*} \mathrm{D} / \mathrm{Vg}+ \\
(1-\alpha)^{*} \mathrm{R} * \mathrm{D} / \mathrm{Vr}\end{array}$ \\
\hline Chibloodg & $\begin{array}{l}\text { Fraction of feeding contacts from chickens in bugs collected in } \\
\text { spring }\end{array}$ & Chibloodg $=\alpha^{*} \mathrm{R}^{*} \mathrm{C} / \mathrm{Vg}$ \\
\hline Humbloodr & $\begin{array}{l}\text { Fraction of feeding contacts from humans in bugs collected in } \\
\text { summer }\end{array}$ & $\begin{array}{l}\text { Humbloodr }=\alpha^{*} \mathrm{H} / \mathrm{Vr}+(1- \\
\alpha)^{*} \mathrm{H} / \mathrm{Vg}\end{array}$ \\
\hline Dogbloodr & $\begin{array}{l}\text { Fraction of feeding contacts from dogs in bugs collected in } \\
\text { summer }\end{array}$ & $\begin{array}{l}\text { Dogbloodr }=\alpha^{*} \mathrm{R} * \mathrm{D} / \mathrm{Vr}+(1- \\
\alpha)^{*} \mathrm{R} * \mathrm{D} / \mathrm{Vg}\end{array}$ \\
\hline Chibloodr & $\begin{array}{l}\text { Fraction of feeding contacts from chickens in bugs collected in } \\
\text { summer }\end{array}$ & Chibloodr $=(1-\alpha)^{*} \mathrm{R}^{*} \mathrm{C} / \mathrm{Vg}$ \\
\hline Abar & Average age of humans (years) & $\begin{array}{l}\text { Abar }=\operatorname{Sum}\left(\mathrm{a}^{*} \mathrm{Ha}\right) / \mathrm{H} \\
\text { Abar }=16.5 \text { years old }\end{array}$ \\
\hline AI & Average age of infected humans (years) & $\begin{array}{l}\mathrm{AI}=\operatorname{Sum}\left(\mathrm{a}^{*} \mathrm{Nha}\right) / \mathrm{NH} \\
\mathrm{AI}=19.9391 \text { years old }\end{array}$ \\
\hline
\end{tabular}

\section{References}

1. E. H. Bucher, C. J. Schofield, New Scientist 92, 321 (1981).

2. M. A. Miles, Trans. R. Soc. Trop. Med. Hyg. 70, 521 (1977).

3. R. Zeledón, J. E. Rabinovich, Annu. Rev. Entomol. 26, 101 (1981).

4. K. E. Mott et al., Am. J. Trop. Med. Hyg. 27, 1116 (1978).

5. R. E. Gürtler, M. C. Cécere, D. P. Vazquez, R. Chuit, J. E. Cohen, J. Med. Entomol. 33, 15 (1996).

6. R. E. Gürtler, J. E. Cohen, M. C. Cecere, R. Chuit, E. L. Segura, Am. J. Trop. Med. Hyg. 58, 748 (1998).

7. M. C. Cecere, M. B. Castañera, D. M. Canale, R. Chuit, R. E. Gürtler, Pan Am. J. Publ. Health 5, 392 (1999).

8. R. E. Gürtler et al., Am. J. Trop. Med. Hyg. 55, 24 (1996). 
9. D. M. Minter, in American Trypanosomiasis Research, PAHO Sci. Publ. 318 (Washington D.C., 1976), pp. 330-337.

10. C. J. Schofield, Ann. Soc. Belg. Med. Trop. 65 (Suppl. 1), 149 (1985).

11. M. C. Cecere, thesis, University of Buenos Aires (1999).

12. M. C. Cecere, R. E. Gürtler, D. Canale, R. Chuit, J. E. Cohen, Bull. WHO 76, 373 (1998).

13. R. A. Ronderos, J. A. Schnack, J. M. Ghilini, G. R. Spinelli, Ecosur 8, 1 (1981).

14. P. D. Marsden et al., Rev. Inst. Med. Trop. Sao Paulo 21, 13 (1979).

15. J. E. Rabinovich, P. H. Himschoot, Ecol. Model. 52, 249 (1990).

16. R. E. Gürtler, J. E. Cohen, M. C. Cecere, R. Chuit, J. Appl. Ecol. 34, 699 (1997).

17. J. E. Rabinovich, C. Wisnivesky-Colli, N. D. Solarz, R. E. Gürtler, Bull. WHO 68, 737 (1990).

18. J. E. Rabinovich, O. Rossell, in American Trypanosomiasis Research, PAHO Sci. Publ. 318 (Washington, D.C., 1976), pp. 359-369.

19. D. W. Kelly, C. E. Thompson, Parasitology 120, 319 (2000).

20. E. V. Trumper, D. E. Gorla, Trans. R. Soc. Trop. Med. Hyg. 85, 800 (1991).

21. P. Cabeza-Meckert, J. G. Chambo, R. P. Laguens, Agents Chemother. 32, 241 (1988).

22. L. C. Giojalas, S. S. Catalá, S. N. Asin, D. E. Gorla, Trans. R. Soc. Trop. Med. Hyg. 84, 439 (1990).

23. P. H. Himschoot, thesis, University of Buenos Aires (1993).

24. M. B. Castañera, thesis, University of Buenos Aires (1999).

25. T. Sota, M. Mogi, Med. Vet. Entomol. 3, 337 (1989).

26. C. C. Lord, M. E. J. Woolhouse, J. A. P. Heesterbeek, P. S. Mellor, Med. Vet. Entomol. 10, 19 (1996).

27. R. Norman, R. G. Bowers, M. Begon, P. J. Hudson, J. Theor. Biol. 200, 111 (1999).

28. MathWorks Inc., Matlab 5.2 Product Family New Features (Natick, MA, 1998). 